\title{
RNA-Seq Analysis of the Arabidopsis Transcriptome in Pluripotent Calli
}

\author{
Kyounghee Lee ${ }^{1}$, Ok-Sun Park', and Pil Joon Seo ${ }^{1,2, *}$
}

\begin{abstract}
Plant cells have a remarkable ability to induce pluripotent cell masses and regenerate whole plant organs under the appropriate culture conditions. Although the in vitro regeneration system is widely applied to manipulate agronomic traits, an understanding of the molecular mechanisms underlying callus formation is starting to emerge. Here, we performed genome-wide transcriptome profiling of wild-type leaves and leaf explant-derived calli for comparison and identified 10,405 differentially expressed genes (> two-fold change). In addition to the well-defined signaling pathways involved in callus formation, we uncovered additional biological processes that may contribute to robust cellular dedifferentiation. Particular emphasis is placed on molecular components involved in leaf development, circadian clock, stress and hormone signaling, carbohydrate metabolism, and chromatin organization. Genetic and pharmacological analyses further supported that homeostasis of clock activity and stress signaling is crucial for proper callus induction. In addition, gibberellic acid (GA) and brassinosteroid (BR) signaling also participates in intricate cellular reprogramming. Collectively, our findings indicate that multiple signaling pathways are intertwined to allow reversible transition of cellular differentiation and dedifferentiation.
\end{abstract}

\section{INTRODUCTION}

Plant somatic cells can undergo dedifferentiation processes to give rise to pluripotent cell masses, called calli. The dedifferentiated cells are able to regenerate new organs or whole plants (Sugimoto et al., 2010). The remarkable plasticity of cellular differentiation allows plants to optimize their growth and development in response to environmental stimuli and thus overcome their sessile nature (Grafi and Barak, 2015).

\footnotetext{
${ }^{1}$ Department of Bioactive Material Sciences and Research Center of Bioactive Materials, ${ }^{2}$ Department of Chemistry and Research Institute of Physics and Chemistry, Chonbuk National University, Jeonju 561-756, Korea

${ }^{*}$ Correspondence: pjseo1@jbnu.ac.kr
}

Received 23 February, 2016; revised 28 April, 2016; accepted 28 April, 2016; published online 24 May, 2016

Keywords: Arabidopsis, biological process, callus formation, dedifferentiation, RNA-Seq
Several genetic pathways are associated with the cellular dedifferentiation process. Consistent with the observations that two growth promoting hormones, auxin and cytokinin, stimulate callus formation (lkeuchi et al., 2013), hormone signaling pathways are implicated in cellular dedifferentiation. In particular, the AUXIN RESPONSE FACTOR7 (ARF7) and ARF19 proteins regulate several members of the LATERAL ORGAN BOUNDARIES DOMAIN (LBD)/ASYMMETRIC LEAVES2LIKE proteins, including LBD16, LBD17, LBD18, and LBD29, to promote auxin-induced callus formation (Fan et al., 2012; Okushima et al., 2007). In parallel with auxin signaling, cytokinin-responsive type-B ARABIDOPSIS RESPONSE REGULATORs (ARRs) also positively regulate callus formation (Mason et al., 2005).

Reacquisition of cell proliferative activity is an important feature of callus formation, and the hormone signaling pathways are consistently integrated into the cell cycle program. LBD proteins control the E2 PROMOTER BINDING FACTOR a (E2Fa) transcription factor that promotes DNA replication together with DIMERIZATION PARTNER (DP) (Berckmans et al., 2011). Accordingly, overexpression of E2Fa contributes to callus formation in some plant species (Kosugi and Ohashi, 2003). Furthermore, auxin-regulated PROPORZ1 (PRZ1) suppresses KIP-RELATED PROTEIN (KRP) genes encoding cyclindependent kinase (CDK) inhibitors (Sieberer et al., 2003), influencing callus formation. In addition to the auxin signaling pathways, type-B ARRs may also promote the cell cycle by activating cyclins, such as CYCD3s (Argyros et al., 2008). Two APETALA2/ETHYLENE-RESPONSIVE FACTOR (AP2/ERF) transcription factors, ENHANCED SHOOT REGENERATION 1 (ESR1) and ESR2, are involved in cytokinin-dependent callus formation by activating CYCD1;1 and CYCD3;1 (lkeda et al., 2006; Ikeuchi et al., 2013).

Embryonic or meristematic regulators are also involved in callus formation. Ectopic expression of a transcriptional activator of embryogenesis, such as LEAFY COTYLEDON 1 (LEC1), LEC2, or AGAMOUS-LIKE 15 (AGL15), results in embryonic callus formation from somatic cells even on a hormone-free medium (Gaj et al., 2005; Guo et al., 2013; Harding et al., 2003; Thakare et al., 2008). AP2/ERF transcription factors involved in embryo development, including BABY BOOM (BBM) and EMBRYOMAKER (EMK)/AINTEGUMENTA-LIKE5 (AIL5)/ PLETHORA5 (PLT5), also participate in cellular dedifferentiation (Boutilier et al., 2002; Tsuwamoto et al., 2010). In addition, the homeodomain-containing transcription factor WUSCHEL (WUS), a key regulator of meristem homeostasis, plays a role in callus induction (Zuo et al., 2002). 
Notably, the callus has organized structures that resemble lateral root primordia (Sugimoto et al., 2010). Transcriptome analysis indicates that gene expression profiles of the callus tissues are similar to those of lateral root meristem (Sugimoto et al., 2010). Although root developmental pathways are unequivocally associated with cellular dedifferentiation, leaf explants can be used for in vitro callus formation. Therefore, genome-wide massive reprogramming of gene expression is required for callus formation from leaf explants (He et al., 2012), although the molecular basis of the acquisition of pluripotency in leaves remains to be fully elucidated. Here, we performed transcriptional profiling using high-throughput next-generation sequencing (RNA-Seq) to highlight genes that are differentially regulated in leaves and leaf explant-derived calli. Multiple pathways were massively interconnected in the cellular dedifferentiation process, and we confirmed the biological relevance of selected biological processes. Our study provides biological insight into the intricate molecular signaling networks underlying the cellular dedifferentiation process in Arabidopsis.

\section{MATERIALS AND METHODS}

\section{Plant materials and growth conditions}

Arabidopsis thaliana (Columbia-0) seeds were germinated on Murashige and Skoog (MS) medium at $22-23^{\circ} \mathrm{C}$ with a $16-\mathrm{h}$ light/8-h dark photoperiod. The toc1-3 (SALK_203853) (Lee et al., 2016) and Qhai1-1 and Qabi2-2 (Rodrigues et al., 2013) mutants were previously reported.

For callus induction, leaf explants of two-week-old plants were placed on callus-inducing medium (CIM) (B5 medium supplemented with $0.5 \mu \mathrm{g} / \mathrm{ml}$ 2,4-dichlorophenoxyacetic acid [2,4-D] and $0.05 \mu \mathrm{g} / \mathrm{ml}$ kinetin), followed by incubation at $22^{\circ} \mathrm{C}$ in the dark for 2 weeks (Fan et al., 2012). To determine the effects of paclobutrazol (PAC) and brassinolide (BL) on callus formation, $1 \mu \mathrm{M}$ PAC (MB-P5699, MB cell, USA) and $0.1 \mathrm{nM}$ and $1 \mathrm{nM} \mathrm{BL}$ (E1641, Sigma-Aldrich, USA) were added to CIM.

\section{Quantitative real-time $R T-P C R$ analysis}

Total RNA was extracted using TRI agent (TAKARA Bio, Japan) according to the manufacturer's recommendations. Reverse transcription (RT) was performed using Moloney Murine Leukemia Virus (M-MLV) reverse transcriptase (Dr. Protein, Korea) with oligo (dT18) to synthesize first-strand cDNA from $2 \mu \mathrm{g}$ of total RNA. Total RNA samples were pretreated with an RNAsefree DNAse. cDNAs were diluted to $100 \mu \mathrm{l}$ with TE buffer, and 1 $\mu$ of diluted cDNA was used for PCR amplification.

Quantitative RT-PCR reactions were performed in 96-well blocks using the Step-One Plus Real-Time PCR System (Applied Biosystems). The PCR primers used are listed in Supplementary Table 1. The values for each set of primers were normalized relative to EUKARYOTIC TRANSLATION INITIATION FACTOR 4A1 (elF4A) (At3g13920) levels. All RTqPCR reactions were performed in triplicate using total RNA samples extracted from three independent biological replicates. A comparative $\Delta \Delta \mathrm{C}_{\mathrm{T}}$ method was employed to evaluate relative quantities of each amplified product in the samples. The threshold cycle $\left(\mathrm{C}_{\mathrm{T}}\right)$ was automatically determined for each reaction using the instrument software with default parameters. The specificity of each $\mathrm{RT}-\mathrm{qPCR}$ reaction was determined by melting curve analysis of the amplified products using the standard method installed in the system.

mRNA-Seq data

To construct RNA libraries with the TruSeq RNA library kit, $1 \mu \mathrm{g}$ of total RNA was used. The procedure included polyA-selected RNA extraction, RNA fragmentation, random hexamer primed reverse transcription, and 100-nt paired-end sequencing by Illumina HiSeq2000. Libraries were quantified using qPCR according to the qPCR Quantification Protocol Guide and qualified using an Agilent Technologies 2100 Bioanalyzer.

To estimate expression levels, the RNA-Seq reads were mapped to the Arabidopsis reference genome (ftp://ftp. arabidopsis.org/home/tair) using TopHat (Trapnell et al., 2009), which is capable of reporting split-read alignments across splice junctions. Transcript counts were calculated, and the relative transcript abundances were measured in FPKM (Fragments Per Kilobase of exon per Million fragments mapped) using Cufflinks.

\section{Statistical analysis of gene expression levels}

Raw data were calculated as the FPKM of each transcript in each sample using cufflinks software. We excluded transcripts with zeroed FPKM values of more than one for total samples. We added 1 to FPKM values of filtered transcripts to facilitate log2 transformation. Filtered data were transformed logarithmically and normalized using a quantile normalization method. For each transcript, we calculated fold change between case and control. Differentially expressed transcripts were determined by adjusting Ifold change $\geq 2$ of more than at least one of total comparisons.

\section{Gene ontology (GO) term enrichment analysis}

Gene functional annotation analysis for DEG list was performed using the DAVID tool (http://david.abcc.ncifcrf.gov/) (Huang et al., 2009) to understand the biological meanings behind a large list of genes. The DAVID tool provides functional annotation in over 40 annotation categories, including GO terms, proteinprotein interactions, protein functional domains, disease associations, bio-pathways, sequence general features, homologies, gene functional summaries, gene tissue expressions, and literature. In the DAVID annotation system, a modified Fisher Exact $p$ value (EASE score) is adopted to measure geneenrichment in annotation terms. If the EASE Score is lower than 0.05 for the specific GO-term, we interpret that the given gene list is specifically associated with the GO term rather than being due to random chance. All data analysis and visualization of differentially expressed genes were conducted using R 3.1.2 software (www.r-project.org).

\section{Immunoblot analysis}

Harvested plant materials were ground in liquid nitrogen, and total cellular extracts were suspended in sodium dodecyl sulphate-polyacrylamide gel electrophoresis (SDS-PAGE) sample loading buffer. Protein samples were analyzed using SDSPAGE (10\% gels) and blotted onto Hybond- $\mathrm{P}^{+}$membranes (Amersham-Pharmacia). Proteins were immunologically detected using anti-H3K4me3 or anti- H3K36me3 antibodies (Millipore, USA).

\section{RESULTS AND DISCUSSION}

\section{Validation of RNA-Seq analysis}

To identify the transcriptional networks controlled during the cellular dedifferentiation process, we compared the transcriptomic profiles of $3^{\text {rd }}$ leaves of two-week-old wild-type plants and leaf explant-derived calli using genome-wide RNA sequencing (RNASeq) (Fig. 1A). RNA was isolated from a pooled callus sample rather than from biological replicates, which is similar to the 
A
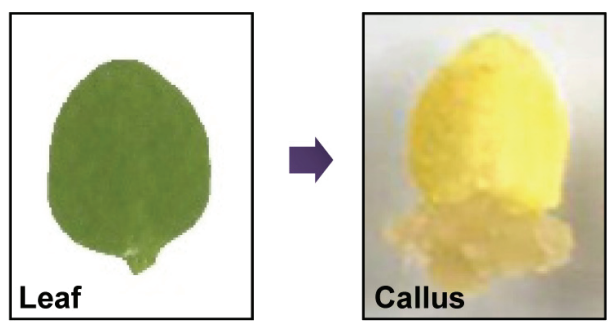

B
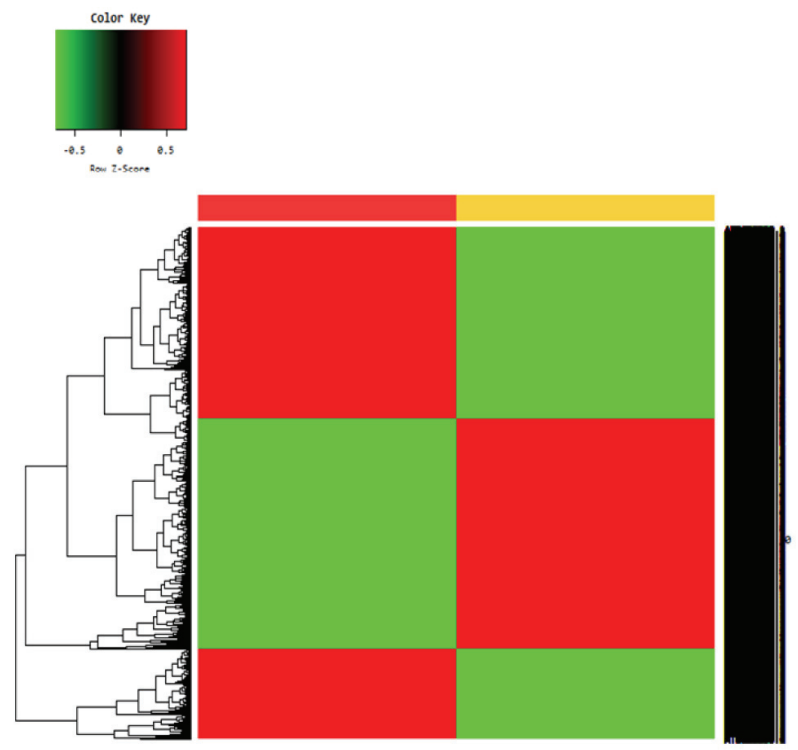

Fig. 1. RNA-Seq analysis to identify genes differentially expressed in leaves and calli. (A) Leaf explant-derived callus formation. Representative samples used for RNA-Seq analysis were photographed. Leaf explants from third-leaves of two-week-old wild-type Columbia-0 (Col-0) plants were used to induce calli on callusinducing medium (CIM). (B) Two-way hierarchical clustering heatmap. In total, 24,497 transcripts were normalized using Z-score calculation. Differentially expressed transcripts in leaves (yellow bar) and calli (red bar) were clustered. The color key in the top lefthand corner is for colors in the heat map.

averaged samples used for other studies (Holmes-Davis et al., 2005; Niederhuth et al., 2013). We sequenced the libraries on Illumina HiSeq 2000 and generated a total of 24,261,279 high quality reads. The reads were aligned onto the Arabidopsis reference genome assembly (TAIR10) (Supplementary Table 2).

We found 5,708 up-regulated and 4,697 down-regulated genes in calli relative to leave tissues ( $>$ two-fold change in expression) (Supplementary Tables 3 and 4). These genes were divided into two groups using a hierarchical clustering algorithm (Fig. 1B). Among the genes differentially expressed, well-known positive regulators of callus formation, such as AGL15, ARF19, EMK/AIL5/PLT5, LBD18 and RESPONSE REGULATOR 1 (RR1) (Fan et al., 2012; Sakai et al., 2001; Thakare et al., 2008; Tsuwamoto et al., 2010), were included (Supplementary Table 5), supporting the reliability of our analysis.

To validate the gene expression profiles revealed by RNASeq analysis, we performed quantitative real-time RT-PCR
(RT-qPCR) analysis and examined transcript accumulation of 10 randomly selected genes: AT1G01060, AT2G43010, AT2 G45420, AT2G46830, AT3G20810, AT4G20400, AT4G25470, AT4G33470, AT4G37650, and AT5G13790 (Supplementary Tables 3 and 4). As expected, all genes examined were significantly and differentially expressed in calli (Supplementary Fig. 1 ), which is highly consistent with our estimates using RNA-Seq data.

Various biological processes involved in callus formation The global transcriptome changes in callus samples were further categorized based on their GO that suggest predicted or experimentally defined biological processes, molecular functions, and cellular components. Functional categorization of differentially regulated genes revealed that a wide variety of biological processes are associated with cellular reprogramming. In particular, functional annotations were highly enriched for functions related to protein metabolism and plant responses to biotic and abiotic stresses (Fig. 2A). Molecular functions were also widely distributed with particular enrichment related to biological macromolecule binding and transferase activity (Fig. 2B). While cellular component is largely a prediction, enriched cellular component terms included categories related to nucleus and chloroplast (Fig. 2C). Since leaf characteristics disappear during callus formation, it seems supportive of our analysis. Because biological process categories are typically derived empirically and thus tend to be more stringent, we focused on biological functions enriched in calli to understand the molecular mechanism underlying cellular dedifferentiation.

\section{Leaf development}

Although callus formation is known to follow lateral root developmental programs (Sugimoto et al., 2010), pluripotent cells can also be produced from aerial explants. Reactivation of root developmental pathways and/or genetic elimination of aerial features may accompany cellular dedifferentiation from leaf somatic cells. In support of this, the core components of Polycomb Repressive Complex 2 (PRC2), including CURLY LEAF (CLF) and SWINGER (SWN) that catalyze histone H3 lysine 27 trimethylation ( $\mathrm{H} 3 \mathrm{~K} 27 \mathrm{me} 3)$ during callus formation to suppress leaf characteristics, contribute to stimulating callus formation from leaf explants (He et al., 2012).

We found 149 genes involved in leaf development were down-regulated in calli compared with leaves (Supplementary Table 6). In particular, predominant members of key transcription factor families involved in leaf development were included, including TEOSINTE BRANCHED1, CYCLOIDEA, AND PCF (TCP), BEL1-LIKE HOMEODOMAIN (BLH), and NGATHA (NGA) (Kumar et al., 2007; Nag et al., 2009; Trigueros et al., 2009). The Arabidopsis genome contains 13 class I and 11 class II TCP genes (Koyama et al., 2010; Tao et al., 2013). Consistent with the fact that the class II members have been characterized as major regulators of leaf development (Tao et al., 2013), expression levels of TCP2, TCP3, TCP4, and TCP10 genes were reduced in calli (Table 1 and Supplementary Table 6). In addition, BLH2/SAWTOOTH1 (SAW1) and BLH4/SAW2, which establish shoot architecture together with SHOOTMERISTEMLESS (STM) and BREVIPEDICELLUS (BP) (Kumar et al., 2007), were also down-regulated (Table 1 and Supplementary Table 6). NGA transcription factors are also implicated in leaf development, as shown in quadruple nga1 nga2 nga3 nga4 mutants that display abnormal leaf development (Trigueros et al., 2009), and accordingly, the NGA2 and NGA3 genes were significantly repressed in calli (Table 1 and 
A

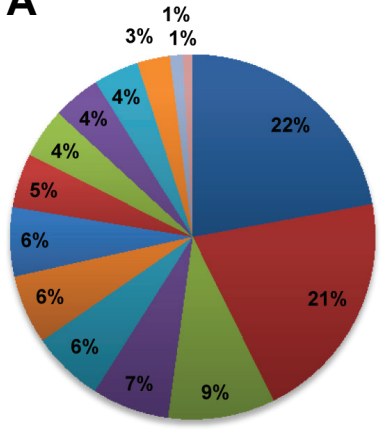

- Other cellular processes

- Other metabolic processes

- Unknown biological processes

-Protein metabolism

Response to stress

Response to abiotic or biotic stimulus

-Other biological processes

-Developmental processes

- Cell organization and biogenesis

- Transport

- Transcription (DNA-dependent)

- Signal transduction

DNA or RNA metabolism

Electron transport or energy pathways

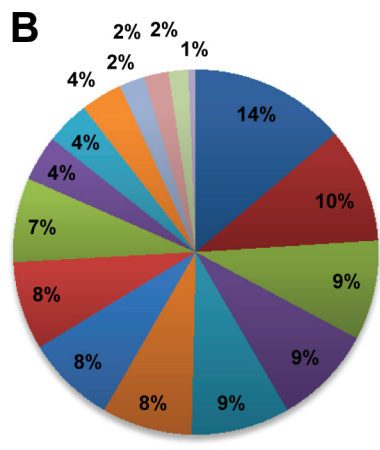

Unknown molecular function

Protein binding

- Other binding

- Transferase activity

DNA or RNA binding

Other enzyme activity

Other binding

Nucleotide binding

-Transcription factor activity

-Transporter activity

Kinase activity

- Kinase activity

-Other molecular functions

Nucleic acid binding

-Receptor binding or activity

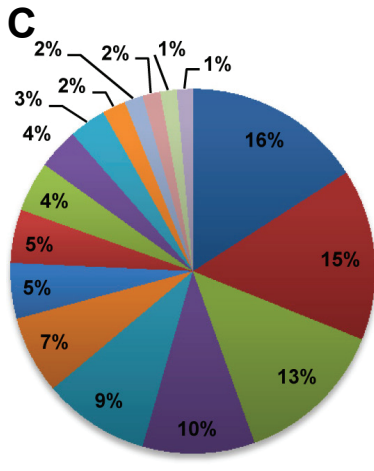

- Other cytoplasmic component

-Nucleus

- Other intracellular components

-Chloroplast

- Other membranes

- Plasma membran

- Mitochondria

- Plastid

Cytosol

Extracellular

- Other cellular components

Golgi apparatus

ER

-Cell wall

Ribosome

- Unknown cellular components

Fig. 2. GO plant term enrichment. Genes differentially expressed in calli were categorized and annotated based on biological processes (A), molecular functions (B), and cellular components (C).

Supplementary Table 6).

In addition, a significant portion of genes related to leaf identity, lateral organ meristem establishment, chloroplast development, and photosynthesis was substantially repressed in calli (Supplementary Table 6). These results indicate that genetic components establishing leaf identity are suppressed during callus formation to turn off differentiated cell identity.

\section{Circadian clock}

The circadian clock generates the biological rhythm with a period of $\sim 24 \mathrm{~h}$, synchronizing internal processes with environmental cycles. Correct matching of the circadian period with external cues ensures plant fitness, and consistently, nearly all stages of plant development are under the control of the circadian clock (Yakir et al., 2007). Previous findings have shown that a
A
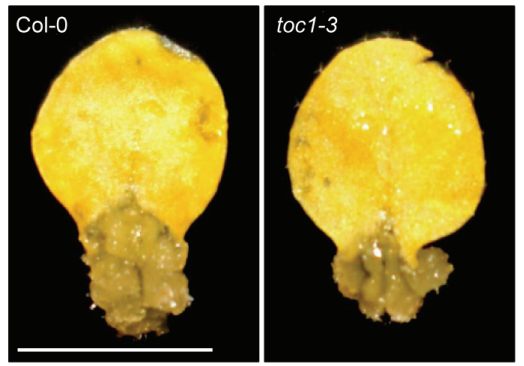

B

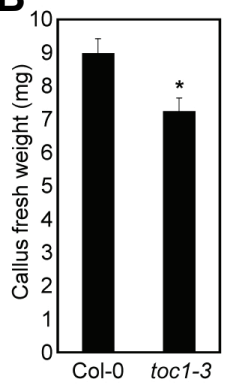

Fig. 3. Callus formation capability of the toc1-3 mutant. (A) Callus formation. Leaf explants from third-leaves of two-week-old plants were used to induce calli on CIM $(n>30)$. Plates were incubated for 2 weeks under continuous dark conditions and photographed. Scale bars $=5 \mathrm{~mm}$. (B) Fresh weight measurement. Thirty calli were collected to measure fresh weight. Bars indicate the standard error of the mean. Statistically significant differences between wildtype and toc1-3 mutant are indicated by asterisks (Student's $t$-test, $\left.{ }^{*} P<0.05\right)$.

biological rhythm retains in dedifferentiated Arabidopsis protoplast cells (Nakamichi et al., 2004), suggesting a possible role of the circadian clock in cellular differentiation and dedifferentiation.

We found that at least 138 circadian-related genes were differentially regulated in calli (Supplementary Table 7). Notably, many core clock oscillator components in multiple-interlocked circadian feedback loops, including CIRCADIAN CLOCK-ASSOCIATED 1 (CCA1), LATE ELONGATED HYPOCOTYL (LHY), TIMING OF CAB EXPRESSION 1/ PSEUDO-RESPONSE REGULATOR 1 (TOC1/PRR1), EARLY FLOWERING 3 (ELF3), and ELF4, were included in the lists (Table 1 and Supplementary Table 7 ), supporting the requirements of endogenous circadian networks in the establishment and maintenance of pluripotency.

To estimate the relevance of circadian functions in cellular dedifferentiation, we employed a genetic mutant that harbors a defect in the core clock oscillator TOC1, the toc1-3 mutant, and compared its callus formation rate with wild-type. Callus formation was reduced when derived from toc1-3 mutant leaves (Fig. 3). Fresh weight measurements revealed that the toc1-3 mutant showed a 1.2-fold reduction in callus formation capability (Fig. 3). Considering that leaf explants were placed in darkness for 2 weeks to induce calli, it is unlikely that circadian oscillation persists over the period of cellular reprogramming. Instead of circadian fluctuations, clock-controlled signaling networks might be associated with robust callus formation.

\section{Plant responses to biotic stress}

Since plants are sessile organisms, they have evolved elaborate dedifferentiation abilities to ensure developmental plasticity. Biotic stress occasionally stimulates cell fate reprogramming. For instance, Agrobacterium tumefaciens causes crown gall tumor in a wide-range of host plants (Gohlke and Deeken, 2014). Infection with Rhizobium meliloti also leads to cellular dedifferentiation in Medicago root cortical cells (Charon et al., 1997). A few genetic components responsible for biotic stressinduced cellular dedifferentiation have been identified. The wounding-responsive AP2/ERF transcription factor WOUNDINDUCED DEDIFFERENTIATION 1 (WIND1) and its homologs, WIND2, WIND3 and WIND4, are possible candidates for 
Table 1. Transcript profiles of key genes involved in selected biological processes. Genes differentially expressed in calli were functionally categorized according to gene ontology (GO) at Arabidopsis Information Resource. The key components of selected biological processes were shown to estimate their biological relevance. Genes were rank ordered in each category by fold change $(F C)$ in expression.

\begin{tabular}{|c|c|c|}
\hline Transcript & Description & Calli/Leaves (FC) \\
\hline \multicolumn{3}{|l|}{ Leaf development } \\
\hline AT4G18390.1 & TEOSINTE BRANCHED 1, CYCLOIDEA AND PCF TRANSCRIPTION FACTOR 2 (TCP2) & -2.39 \\
\hline AT3G61970.1 & NGATHA2 (NGA2) & -3.88 \\
\hline AT1G53230.1 & TCP FAMILY TRANSCRIPTION FACTOR 3 (TCP3) & -4.28 \\
\hline AT1G01030.1 & NGATHA3 (NGA3) & -5.29 \\
\hline AT3G15030.1 & TCP FAMILY TRANSCRIPTION FACTOR 4 (TCP4) & -7.81 \\
\hline AT2G23760.1 & BEL1-LIKE HOMEODOMAIN 4 (BLH4) & -10.58 \\
\hline AT4G36870.1 & BEL1-LIKE HOMEODOMAIN 2 (BLH2) & -14.27 \\
\hline AT2G31070.1 & TCP DOMAIN PROTEIN 10 (TCP10) & -30.50 \\
\hline \multicolumn{3}{|l|}{ Circadian clock } \\
\hline AT5G61380.1 & TIMING OF CAB EXPRESSION 1 (TOC1) & 9.16 \\
\hline AT2G25930.1 & EARLY FLOWERING 3 (ELF3) & 3.64 \\
\hline AT2G40080.1 & EARLY FLOWERING 4 (ELF4) & 3.23 \\
\hline AT2G46830.1 & CIRCADIAN CLOCK-ASSOCIATED 1 (CCA1) & -18.11 \\
\hline AT1G01060.1 & LATE ELONGATED HYPOCOTYL (LHY) & -25.42 \\
\hline \multicolumn{3}{|l|}{ Response to virus } \\
\hline АT3G03300.2 & DICER-LIKE 2 (DCL2) & 2.67 \\
\hline AT2G27040.1 & ARGONAUTE 4 (AGO4) & 2.13 \\
\hline AT1G48410.2 & ARGONAUTE 1 (AGO1) & 2.12 \\
\hline \multicolumn{3}{|c|}{ Response to bacterium } \\
\hline AT3G52430.1 & PHYTOALEXIN DEFICIENT 4 (PAD4) & 11.79 \\
\hline AT3G48090.1 & ENHANCED DISEASE SUSCEPTIBILITY 1 (EDS1) & 8.15 \\
\hline AT4G31800.1 & WRKY DNA-BINDING PROTEIN 18 (WRKY18) & 7.14 \\
\hline AT5G45110.1 & NPR1-LIKE PROTEIN 3 (NPR3) & 7.03 \\
\hline AT3G56400.1 & WRKY DNA-BINDING PROTEIN 70 (WRKY70) & 4.23 \\
\hline AT5G65210.1 & TGACG SEQUENCE-SPECIFIC BINDING PROTEIN 1 (TGA1) & 3.05 \\
\hline AT2G25000.1 & WRKY DNA-BINDING PROTEIN 60 (WRKY60) & 2.55 \\
\hline AT4G31550.2 & WRKY DNA-BINDING PROTEIN 11 (WRKY11) & 2.29 \\
\hline AT2G24570.1 & WRKY DNA-BINDING PROTEIN 17 (WRKY17) & 2.24 \\
\hline AT4G23810.1 & WRKY DNA-BINDING PROTEIN 53 (WRKY53) & 2.20 \\
\hline AT1G22070.1 & TGA1A-RELATED GENE 3 (TGA3) & 2.02 \\
\hline \multicolumn{3}{|c|}{ Response to osmotic stress } \\
\hline AT5G63650.1 & SNF1-RELATED PROTEIN KINASE 2.5 (SNRK2.5) & 37.84 \\
\hline AT5G66880.1 & SNF1-RELATED PROTEIN KINASE 2.3 (SNRK2.3) & 11.29 \\
\hline AT3G50500.1 & SNF1-RELATED PROTEIN KINASE 2.2 (SNRK2.2) & 7.84 \\
\hline AT1G49720.1 & ABSCISIC ACID RESPONSIVE ELEMENT-BINDING FACTOR 1 (ABF1) & 5.19 \\
\hline AT5G59220.1 & HIGHLY ABA-INDUCED PP2C GENE 1 (HAl1) & 3.58 \\
\hline AT4G33950.2 & OPEN STOMATA 1 (OST1) & 3.32 \\
\hline AT4G26080.1 & ABA INSENSITIVE 1 (ABI1) & 2.05 \\
\hline \multicolumn{3}{|c|}{ Response to oxidative stress } \\
\hline AT1G08830.1 & COPPER/ZINC SUPEROXIDE DISMUTASE 1 (CSD1) & 4.97 \\
\hline AT2G48150.1 & GLUTATHIONE PEROXIDASE 4 (GPX4) & 4.74 \\
\hline AT2G43350.1 & GLUTATHIONE PEROXIDASE 3 (GPX3) & 3.88 \\
\hline AT1G20630.1 & CATALASE 1 (CAT1) & 3.72 \\
\hline AT1G63460.1 & GLUTATHIONE PEROXIDASE 8 (GPX8) & 3.57 \\
\hline AT4G11600.1 & GLUTATHIONE PEROXIDASE 6 (GPX6) & 2.38 \\
\hline AT3G10920.2 & MANGANESE SUPEROXIDE DISMUTASE 1 (MSD1) & -2.00 \\
\hline AT4G35000.1 & ASCORBATE PEROXIDASE 3 (APX3) & -2.17 \\
\hline AT5G23310.1 & FE SUPEROXIDE DISMUTASE 3 (FSD3) & -2.20 \\
\hline AT4G32320.1 & ASCORBATE PEROXIDASE 6 (APX6) & -3.13 \\
\hline AT1G07890.6 & ASCORBATE PEROXIDASE 1 (APX1) & -3.19 \\
\hline AT5G18100.2 & COPPER/ZINC SUPEROXIDE DISMUTASE 3 (CSD3) & -5.36 \\
\hline AT2G31570.1 & GLUTATHIONE PEROXIDASE 2 (GPX2) & -6.78 \\
\hline AT4G25100.1 & FE SUPEROXIDE DISMUTASE 1 (FSD1) & -7.89 \\
\hline AT2G25080.1 & GLUTATHIONE PEROXIDASE 1 (GPX1) & -16.83 \\
\hline AT4G31870.1 & GLUTATHIONE PEROXIDASE 7 (GPX7) & -17.40 \\
\hline AT4G35090.2 & CATALASE 2 (CAT2) & -42.58 \\
\hline AT4G09010.1 & ASCORBATE PEROXIDASE 4 (APX4) & -177.58 \\
\hline
\end{tabular}

(continued) 
Table 1.

\begin{tabular}{llr}
\hline Transcript & Description & Calli/Leaves (FC) \\
\hline Polysaccharide metabolic process & 11.23 \\
AT4G09020.1 & ISOAMYLASE 3 (ISA3) & 2.63 \\
AT1G10760.1 & A-GLUCAN WATER, DIKINASE/STARCH EXCESS 1 (GWD1/SEX1) & 2.02 \\
AT5G26570.2 & PHOSPHOGLUCAN WATER DIKINASE (PWD) & -2.11 \\
AT5G64740.1 & CELLULOSE SYNTHASE 6 (CESA6) & -5.31 \\
AT4G39350.1 & CELLULOSE SYNTHASE 2 (CESA2) & -8.84 \\
AT5G09870.1 & CELLULOSE SYNTHASE 5 (CESA5) & 10.63 \\
Gibberellin signaling & & 6.56 \\
AT5G17490.1 & RGA-LIKE PROTEIN 3 (RGL3) & 3.43 \\
AT3G63010.1 & GA INSENSITIVE DWARF1B (GID1B) & 3.42 \\
AT3G03450.1 & RGA-LIKE 2 (RGL2) & 2.86 \\
AT3G05120.1 & GA INSENSITIVE DWARF1A (GID1A) & 2.58 \\
AT1G66350.1 & RGA-LIKE 1 (RGL1) & \\
AT5G27320.1 & GA INSENSITIVE DWARF1C (GID1C) & 2.34 \\
Brassinosteroid signaling & 2.20 \\
AT4G39400.1 & BRASSINOSTEROID INSENSITIVE 1 (BRI1) & \\
AT4G33430.1 & BRI1-ASSOCIATED RECEPTOR KINASE (BAK1) & 4.73 \\
Chromatin organization & & 3.20 \\
AT1G26760.1 & SET DOMAIN PROTEIN 35 (SDG35) & 2.66 \\
AT5G24330.1 & ARABIDOPSIS TRITHORAX-RELATED PROTEIN 6 (ATXR6) \\
AT1G76710.1 & SET DOMAIN GROUP 26 (SDG26) \\
AT5G09790.2 & ARABIDOPSIS TRITHORAX-RELATED PROTEIN 5 (ATXR5) & 2.61 \\
\hline
\end{tabular}

the regulation of callus formation upon exposure to pathogens or herbivory (Iwase et al., 2011).

In addition to the biotic stress-stimulated cellular dedifferentiation process (Grafi, 2004), calli are known to retain a high protective ability against various microbial invaders, suggesting intimate bidirectional regulation. Our RNA-Seq analysis showed that pivotal defense genes were up-regulated in calli. RNA silencing is a representative antiviral defense mechanism in plants. DICER-LIKE (DCL) RNase III enzymes are primarily responsible for catalyzing RNA processing (Henderson et al., 2006). Arabidopsis has four DICER-like proteins, DCL1-DCL4, with functional specificity. Among them, DCL2 is mainly responsible for the generation of virus-derived siRNAs (Gasciolli et al., 2005). Small RNA duplexes are then incorporated into RNAinduced silencing complex (RISC) to target viral RNA for processing (Pumplin and Voinnet, 2013). A central component of RISC complex is the ARGONAUTE (AGO) protein, and in Arabidopsis, ten AGO proteins (AGO1-AGO10) are possible candidates for RISC formation (Garcia-Ruiz et al., 2015). Our RNASeq analysis revealed that 60 virus-responsive genes, including DCL2, AGO1 and AGO4, showed higher expression in calli than in leaves (Table 1 and Supplementary Table 8).

Plants have also developed strong innate immunity against bacterial pathogens. As a first layer of defense, plants activate pathogen-associated molecular pattern (PAMP)-triggered immunity (PTI) upon recognition of PAMPs by the plant pattern recognition receptor (PRR) (Kim et al., 2008; Nicaise et al., 2009). Signaling cascades built-up with MITOGENACTIVATED PROTEIN KINASES (MAPKS), CALCIUMDEPENDENT PROTEIN KINASES (CDPKs), and WRKY transcription factors are subsequently activated (Eulgem and Somssich, 2007; Rasmussen et al., 2012). In addition, plants have a second barrier to pathogen invasion, which stimulates effector-triggered immunity (ETI). Pathogen effector molecules are sensed by various plant resistance $(R)$ proteins, and the recognition triggers hypersensitive response $(\mathrm{HR})$ and sys- temic acquired resistance (SAR) mediated by SA biosynthesis (Dempsey et al., 2011). Notably, 76 bacterial-responsive genes that mediate PTI and/or ETI, such as WRKY11, WRKY17, WRKY18, WRKY53, WRKY60, WRKY70, ENHANCED DISEASE SUSCEPTIBILITY 1 (EDS1), and PHYTOALEXIN DEFICIENT 4 (PAD4), were up-regulated in calli (Table 1 and Supplementary Table 9). Accordingly, SA signaling genes, such as TGACG SEQUENCE-SPECIFIC BINDING PROTEIN 1 (TGA1), TGA3, and NPR1-LIKE PROTEIN 3 (NPR3), were also influenced (Table 1 and Supplementary Table 9), possibly contributing to maintaining disease-free dedifferentiated cells.

\section{Plant responses to abiotic stress}

Environmental stress stimulates cellular dedifferentiation (Grafi et al., 2011). Transcriptome profiling has revealed that differentiated organs experiencing environmental challenges, such as drought and continuous dark, have gene expression profiles similar to those of dedifferentiated protoplast cells (Grafi et al., 2011). Consistently, stress-related genes form the molecular signature for pluripotent animal cells (Ramalho-Santos et al., 2002). These observations suggest that abiotic stressresponsive transcription factors may play a key role in cellular reprogramming. For example, the NAC DOMAINCONTAINING PROTEIN 2 (NAC2) transcription factor is not only responsive to abiotic stresses (Nuruzzaman et al., 2013) but also regulates meristem proliferation activity (Florentin et al., 2013). Thus, NAC2 acts with potential molecular crosstalk of abiotic stress and stem cell signaling and contributes to the reacquisition of stem cell fate in Arabidopsis somatic cells under unfavorable environmental conditions (Florentin et al., 2013).

We found that a significant number of abiotic stress- and/or abscisic acid (ABA)-responsive genes are differentially expressed in calli (Supplementary Table 10). Osmotic stress is largely dependent on $\mathrm{ABA}$, and consistently, osmotic stress primarily influences genes encoding $A B A$ sensory and up- 

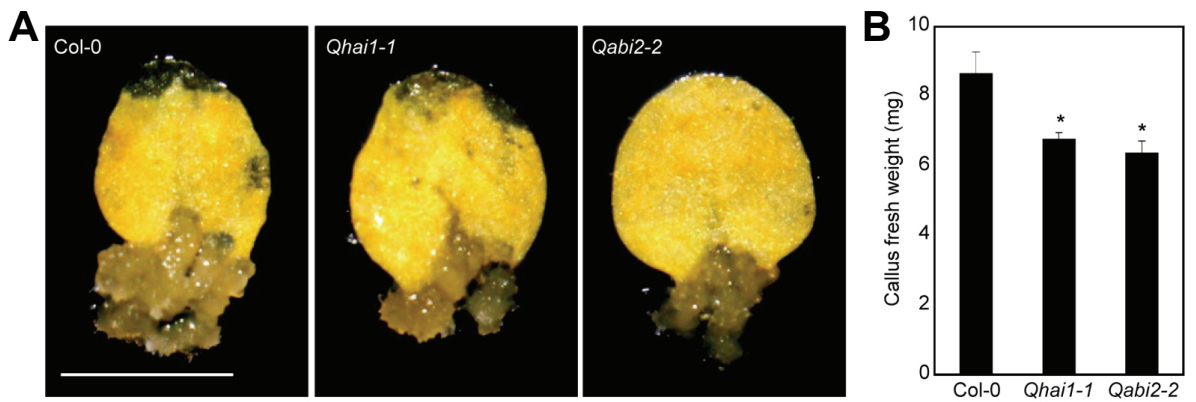

Fig. 4. Callus formation of $A B A$ signaling mutants. (A) Callus formation. Leaf explants from the third leaves of two-week-old plants were used to induce calli on CIM $(n>30)$. Plates were incubated for 2 weeks under continuous dark conditions and photographed. Scale bars $=5 \mathrm{~mm}$. (B) Fresh weight measurement. Thirty calli were collected to measure fresh weight. Bars indicate the standard error of the mean. Statistically signifi-

cant differences between wild-type and mutants are indicated by asterisks (Student's $t$-test, ${ }^{\star} P<0.05$ ).

stream components, such as REGULATORY COMPONENT OF ABA RECEPTORs (RCARs), Type $2 \mathrm{C}$ protein phosphatases (PP2Cs), SNF1-related protein kinase 2s (SnRK2s), and abscisic acid-responsive element-binding factors (ABFs) (Chan, 2012). Notably, 152 osmotic stress-responsive genes were upregulated in calli (Supplementary Table 10), and most notably ABA-INSENSITIVE 1 (ABI1), ABF1, HIGHLY ABA-INDUCED1 (HAl1), SnRK2.2, SnRK2.3, SnRK2.5, and SnRK2.6/OPEN STOMATA 1 (OST1) (Table 1 and Supplementary Table 10). Given that osmotic stress induces somatic embryogenesis in Arabidopsis (Ikeda-Iwai et al., 2003), these genes might play a role in switching cell fates under environmentally unfavorable conditions.

To address whether the ABA signaling genes are indeed involved in switching cell fates, we employed two quadruple mutants, Qhai1-1 (lacking HAl1, PP2CA, HOMOLOGY TO ABI1 (HAB1), and $A B / 1$; Rodrigues et al., 2013) and Qabi2-2 (lacking $A B I 2, P P 2 C A, H A B 1$, and $A B / 1$; Rodrigues et al., 2013), and induced callus formation on CIM. Both quadruple mutants exhibited decreased callus formation capability relative to wildtype plants (Fig. 4), indicating that ABA signaling homeostasis is necessary for proper callus formation. While further detailed analysis is required to unravel the underlying molecular mechanisms, ABA upstream regulators might be a molecular linker of stress adaptation and cellular dedifferentiation.

Reactive oxygen species (ROS) are important signaling molecules in stress responses and cellular differentiation. In addition to their stress-responsive defense functions (Tsukagoshi et al., 2010), ROS also regulate dedifferentiation as exemplified by the promotive effects of hydrogen peroxide $\left(\mathrm{H}_{2} \mathrm{O}_{2}\right)$ on cellular dedifferentiation in Lycium barbarum (Cui et al., 2002). Optimal balances of ROS accumulation are achieved by multiple antioxidative systems. Enzymatic detoxification is catalyzed by superoxide dismutase (SOD), ascorbate peroxidase (APX), catalase (CAT), glutathione peroxidase (GPX), and peroxiredoxin (PRX) (Caverzan et al., 2012). The Arabidopsis genome encodes seven SOD proteins classified depending on their metal cofactors: three $\mathrm{Cu} / \mathrm{Zn}$ SOD proteins [COPPER/ZINC SUPEROXIDE DISMUTASE 1 (CSD1), CSD2, CSD3], three Fe SOD proteins [Fe SUPEROXIDE DISMUTASE 1 (FSD1), FSD2 and FSD3], and one Mn SOD protein [MANGANESE SUPEROXIDE DISMUTASE 1 (MSD1)] (Xing et al., 2013). There are also nine $A P X(A P X 1-9)$, three $C A T$ (CAT1-3) and eight GPX (GPX1-8) genes (Caverzan et al., 2012; Passaia et al., 2014; Xing et al., 2007). In calli, CSD1, CAT1, GPX3, GPX4, $G P X 6$ and $G P X 8$ were up-regulated, whereas $A P X 1, A P X 3$, APX4, APX6, CAT2, CSD3, FSD1, FSD3, GPX1, GPX2, GPX7 and MSD1 were repressed (Table 1 and Supplementary Table
11). Furthermore, many peroxidase and reductase genes were also differentially regulated (Supplementary Table 11), supporting the importance of ROS homeostasis during cellular dedifferentiation.

While it seems clear that abiotic stress triggers cell fate changes, the molecular components responsible for the process are largely unknown. The genes that we proposed in this study might play a role as molecular linkers of stress-induced cellular dedifferentiation. Future studies will also provide biological insights as to why plants dedifferentiate in suboptimal conditions and how pluripotent cells improve plant adaptation ability to environmental challenges.

\section{Carbohydrate metabolism}

The plant cell wall is an important cellular structure that provides structural support and maintains cellular differentiation states (Keegstra, 2010). In support of this, treatments with cell wall-degrading enzymes produce dedifferentiated protoplast cells (Grafi, 2004). Moreover, endo-1,4- $\beta$-d-glucanase KORRIGAN 1/TUMOROUS SHOOT DEVELOPMENT 1 (KOR1/TSD1), which is involved in cellulose synthesis, also participates in the maintenance of somatic cell identity. The tsd 1 loss-of-function mutants display callus-like structures with reduced cell wall formation on a hormone-free medium (Frank et al., 2002).

The plant cell wall is composed of cellulose, hemicellulose lignin, and pectin. In particular, cellulose, a linear chain of $\beta$ (14)-linked D-glucose, is the main component of the cell wall and comprises approximately $50 \%$ of the total cell wall mass (Endler and Persson, 2011). In Arabidopsis, 10 CELLULOSE SYNTHASE (CESA) proteins (CESA1-CESA10) are supposed to catalyze cellulose synthesis (Endler and Persson, 2011). Here, we found that 29 genes involved in polysaccharide metabolism, including CESA2, CESA5, and CESA6, were downregulated in calli (Table 1 and Supplementary Table 12). The genes encoding hemicellulose-metabolizing enzymes, such as xyloglucan endotransglucosylases/hydrolases (XTHs), were also included (Supplementary Table 12).

The storage polysaccharide starch is the energy source in plants (Kötting et al., 2005). Starch is converted into simple soluble sugars by the action of hydrolytic enzymes, such as amylases (Smith et al., 2005; Streb et al., 2012). In Arabidopsis, additional proteins that stimulate starch metabolism have been identified; $\alpha$-GLUCAN WATER, DIKINASE/STARCH EXCESS 1 (GWD1/SEX1) and PHOSPHOGLUCAN, WATER DIKINASE (PWD), which phosphorylate C6 and C3-position of glucosyl residues, respectively, catalyze starch hydrolysis (Santelia et al., 2011). Notably, ISOAMYLASE 3 (ISA3), GWD1, and 
A
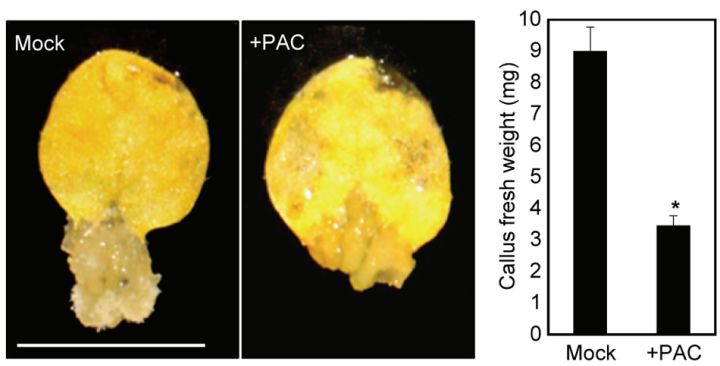

B

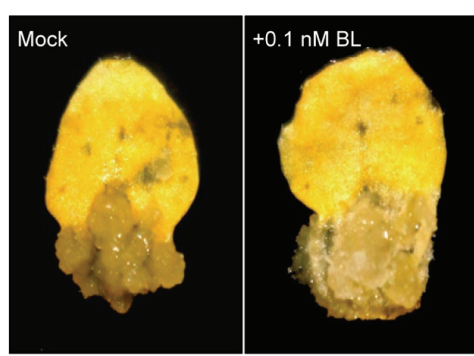

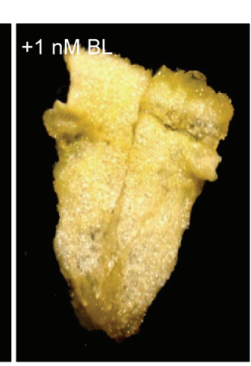

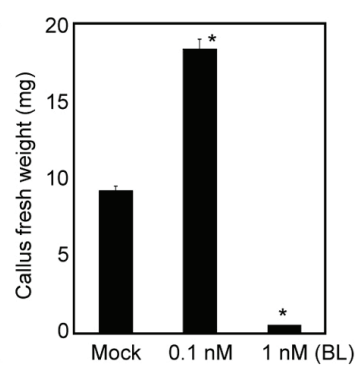

Fig. 5. Effects of $P A C$ and $B L$ on callus formation. Leaf explants from third-leaves of two-week-old wild-type plants were used to induce calli on CIM in the presence of 1 $\mu \mathrm{M}$ paclobutrazol (PAC) $(\mathrm{A})$ and $0.1 \mathrm{nM}$ and $1 \mathrm{nM}$ brassinolide (BL) (B). Plates were incubated for 2 weeks and photographed (left panels). Thirty calli were collected to measure fresh weight. Bars indicate the standard error of the mean. Statistically significant differences compared with the mock-treated sample are indicated by asterisks (Student's $t$-test, ${ }^{*} P$ $<0.05$ ) (right panels).
$P W D$ were up-regulated in calli (Table 1 and Supplementary Table 12), whereas many starch synthase genes were repressed (Supplementary Table 12). These results suggest that active cell proliferation requires a sufficient energy supply (Narbonne and Roy, 2006; Skylar et al., 2011), and simple sugar production might drive the cellular dedifferentiation process.

\section{Hormone signaling}

Two phytohormones, auxin and cytokinin, play a crucial role in cellular dedifferentiation in a variety of plant species. Since the coordinated actions of phytohormones are important for various aspects of plant growth and development (Vanstraelen and Benková, 2012), we hypothesized that additional hormones, such as $A B A$, gibberellin (GA), ethylene (ET), brassinosteroid (BR), jasmonic acid (JA), and salicylic acid (SA), may be implicated in callus formation.

Our RNA-Seq analysis revealed that differential expression of $\mathrm{GA}$ and $\mathrm{BR}$ signaling components in calli were noticeable. In particular, 22 GA-related genes, which encode GA metabolic enzymes, GA receptors, and DELLA proteins, were differentially regulated in calli (Table 1 and Supplementary Table 13). Considering that GA shows a functional overlap with auxin in plant growth and development ( $\mathrm{Fu}$ and Harberd, 2003), crosstalk between GA and auxin may underlie the cellular dedifferentiation process. To prove this hypothesis, we employed a potent chemical inhibitor of GA biosynthesis, PAC, and examined its effects on callus formation capability. Treatment with PAC resulted in significant reduction in callus formation ability, which reaches up to approximately $70 \%$, supporting a positive function of GA in cellular dedifferentiation (Fig. 5A).

The plant steroid hormone BR is closely associated with the processes of cell proliferation and differentiation (GonzálezGarcía et al., 2011). The BR biosynthetic gene DWARF7deficient dwarf7-1 mutant consistently displays reduced callus formation, possibly due to reduced cell division (Cheon et al., 2010). Our analysis further supported the previous reports. BR signalling genes, including BRASSINOSTEROID INSENSITIVE 1 (BRI1) and BRI1-ASSOCIATED RECEPTOR KINASE1
(BAK1), were up-regulated in calli (Table 1 and Supplementary Table 14). In addition, we also noticed that several key BR signaling genes, including BRI1-EMS-SUPPRESSOR 1 (BES1) and BRI1 SUPPRESSOR 1 (BRS1), were repressed in calli (Supplementary Table 14). Consistent with the controversial results, opposite effects of BR on callus formation were observed depending on BL concentrations applied. Callus formation was stimulated by low concentration of BL, but inhibited at higher concentration (Fig. 5B). Different modes of BR actions would underlie the balanced cellular reprogramming. Collectively, these results indicate that additional hormones play a supplemental role in inducing callus formation.

\section{Chromatin organization}

Chromatin structure determines gene expression by regulating the accessibility of transcriptional machinery. In higher eukaryotes, global gene expression changes facilitated by epigenetic regulation are associated with cellular differentiation status. For instance, in mammals, differentiated cells have a closed chromatin state, whereas pluripotent dedifferentiated cells have an open chromatin state (Gaspar-Maia et al., 2011).

Genome-wide reorganization of chromatin structure is also crucial for cellular dedifferentiation in plants. The PRC2 and PRC1 complexes coordinate establishment and maintenance of repressive histone mark $\mathrm{H} 3 \mathrm{~K} 27 \mathrm{me} 3$ to control cellular differentiation (Lafos et al., 2011). Mutations in PRC2 or PRC1 components lead to spontaneous callus formation from root explants with ectopic expression of LEC1, LEC2, AGL15, BBM, WUS, and WUSCHEL RELATED HOMEOBOX 5 (WOX5) (Bouyer et al., 2011; Bratzel et al., 2010; Chen et al., 2010).

While H3K27 methylation is involved in callus formation, the relevance of other histone marks in this process remains to be fully elucidated. The activation histone marks, H3K4me2/3 and H3K36me2/3, are associated with higher gene expression (Guo et al., 2010; Yang et al., 2012), and the TRITHORAX GROUP (TrxG) and ABSENT, SMALL OR HOMEOTIC DISCS 1 (ASH1) proteins are responsible for the establishment of the activation marks (He et al., 2013). The Arabidopsis genome 

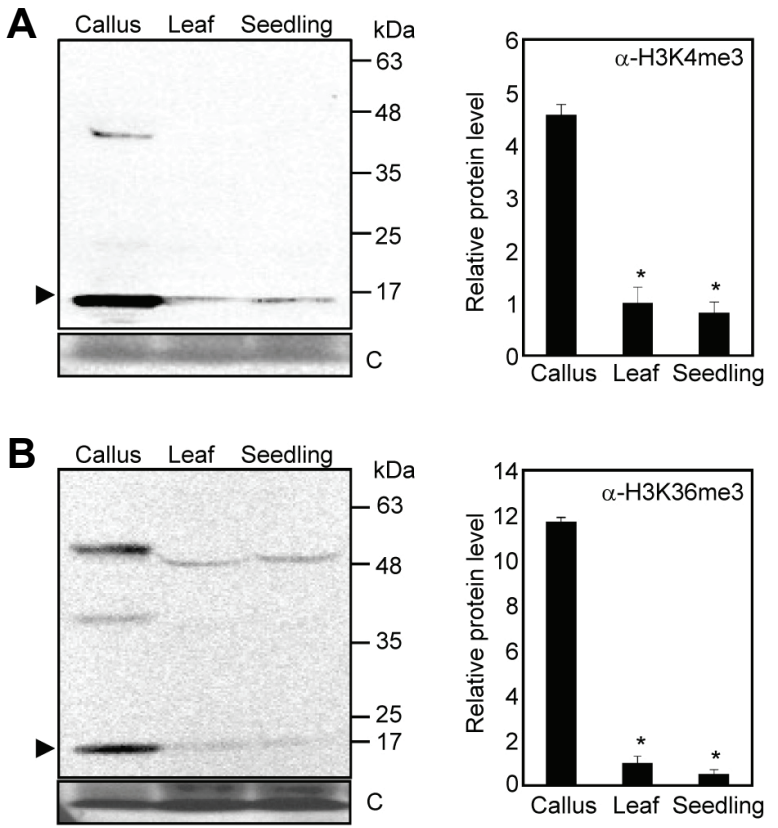

Fig. 6. Total $\mathrm{H} 3 \mathrm{~K} 4 \mathrm{me} 3$ and $\mathrm{H} 3 \mathrm{~K} 36 \mathrm{me} 3$ levels in calli. Leaf explants from third-leaves of two-week-old wild-type plants were used to induce calli on CIM. H3K4me3 (A) and H3K36me3 (B) levels (arrowheads in each) were detected immunologically using the corresponding antibodies. A part of a Coomassie blue-stained gel (C) is shown as a loading control (left panels). Bands from three blots were quantified and averaged using Image $\mathrm{J}$ software. Bars indicate the standard error of the mean. Statistically significant differences between values of leaf and other vegetative tissue samples are indicated by asterisks (Student's $t$-test, ${ }^{\star} P<0.05$ ) (right panels).

has multiple homologs: five ARABIDOPSIS HOMOLOG OF TRITHORAX proteins (ATX1-5), seven ATX-RELATED proteins (ATXR1-7), four ASH1 HOMOLOG proteins (ASHH1-4), and three ASH1-RELATED proteins (ASHR1-3) (Berr et al., 2009). We found that at least 115 genes functionally associated with chromatin remodeling were differentially regulated in calli (Supplementary Table 15). Notably, expression of ATXR1/ SDG35, ATXR5/SDG15, ATXR6/SDG34, and ASHH1/SDG26 were elevated (Table 1 and Supplementary Table 15). Accordingly, immunoblot analysis showed that $\mathrm{H} 3 \mathrm{~K} 4 \mathrm{me} 3$ and H3K36me3 levels were significantly increased in calli relative to vegetative tissues (Fig. 6).

The activation marks established by TrxG and ASH1 counteract, in general, polycomb group (PcG) repression. Given the roles of PcG in cellular dedifferentiation in plants (Bouyer et al., 2011; Bratzel et al., 2010; Chen et al., 2010), it seems meaningful that TrxG functions also underlie the process. In addition, considering the large number of chromatin remodeling genes differentially regulated in calli (Supplementary Table 15), epigenetic regulation is a key regulatory layer for cellular dedifferentiation.

In conclusion, by comparing RNA-Seq read count, we assessed the relative accumulation of RNAs present in the leaf and callus and studied the molecular processes controlling cellular dedifferentiation. We found and confirmed that a variety of biological processes are engaged during callus formation. In particular, molecular components related to shoot development, circadian clock regulation, biotic and abiotic stress responses, nutrient metabolism, and chromatin modification are possibly associated. These results demonstrate the complexity of cellular reprogramming and that the multiple pathways are integrated to facilitate callus formation. Here, we provide valuable initial resources for investigating cellular dedifferentiation in many crop plants and pave the way for improving crop transformation efficiency for genetic engineering.

Note: Supplementary information is available on the Molecules and Cells website (www.molcells.org).

\section{ACKNOWLEDGMENTS}

We would like to thank Dr. Elena Baena-González at Instituto Gulbenkian de Ciência (Oeiras, Portugal) for kindly providing Qhai1-1 and Qabi2-2 seeds. This work was supported by the Next-Generation BioGreen 21 Program (PJ01119204) provided by the Rural Development Administration. K.L. was supported by the BK21 PLUS program in the Department of Bioactive Material Sciences.

\section{REFERENCES}

Argyros, R.D., Mathews, D.E., Chiang, Y.H., Palmer, C.M., Thibault, D.M., Etheridge, N., Argyros, D.A., Mason, M.G., Kieber, J.J., and Schaller, G.E. (2008). Type B response regulators of Arabidopsis play key roles in cytokinin signaling and plant development. Plant Cell 20, 2102-2116.

Berckmans, B., Vassileva, V., Schmid, S.P., Maes, S., Parizot, B., Naramoto, S., Magyar, Z., Alvim Kamei, C.L., Koncz, C., Bögre, L., et al. (2011). Auxin-dependent cell cycle reactivation through transcriptional regulation of Arabidopsis E2Fa by lateral organ boundary proteins. Plant Cell 23, 3671-3683.

Berr, A., Xu, L., Gao, J., Cognat, V., Steinmetz, A., Dong, A., and Shen, W.H. (2009). SET DOMAIN GROUP25 encodes a histone methyltransferase and is involved in FLOWERING LOCUS C activation and repression of flowering. Plant Physiol. 151, 14761485.

Boutilier, K., Offringa, R., Sharma, V.K., Kieft, H., Ouellet, T., Zhang L., Hattori, J., Lium, C.M., van Lammeren, A.A., Miki, B.L., et al. (2002). Ectopic expression of $B A B Y B O O M$ triggers a conversion from vegetative to embryonic growth. Plant Cell 14, 1737-1749.

Bouyer, D., Roudier, F., Heese, M., Andersen, E.D., Gey, D., Nowack, M.K., Goodrich, J., Renou, J.P., Grini, P.E., Colot, V., et al. (2011). Polycomb repressive complex 2 controls the embryoto-seedling phase transition. PLoS Genet. 7, e1002014.

Bratzel, F., López-Torrejón, G., Koch, M., Del Pozo, J.C., and Calonje, M. (2010). Keeping cell identity in Arabidopsis requires PRC1 RING-finger homologs that catalyze H2A monoubiquitination. Curr. Biol. 2, 1853-1859.

Caverzan, A., Passaia, G., Rosa, S.B., Ribeiro, C.W., Lazzarotto, F., and Margis-Pinheiro, M. (2012). Plant responses to stresses: role of ascorbate peroxidase in the antioxidant protection. Genet. Mol. Biol. 35, 1011-1019.

Chan, Z. (2012). Expression profiling of ABA pathway transcripts indicates crosstalk between abiotic and biotic stress responses in Arabidopsis. Genomics 100, 110-115.

Charon, C., Johansson, C., Kondorosi, E., Kondorosi, A., and Crespi, M. (1997). ENOD40 induces dedifferentiation and division of root cortical cells in legumes. Proc. Natl. Acad. Sci. USA 94, 8901-8906.

Chen, D., Molitor, A., Liu, C., and Shen, W.H. (2010). The Arabidopsis PRC1-like ring-finger proteins are necessary for repression of embryonic traits during vegetative growth. Cell Res. 20, 1332-1344.

Cheon, J., Park, S.Y., Schulz, B., and Choe, S. (2010). Arabidopsis brassinosteroid biosynthetic mutant dwarf7-1 exhibits slower rates of cell division and shoot induction. BMC Plant Biol. 10, 270

Cui, K., Li, J., Xing, G., Li, J., Wang, L., and Wang, Y. (2002). Effect of hydrogen peroxide on synthesis of proteins during somatic embryogenesis in Lycium barbarum. Plant Cell Tissue Organ Cult. 68, 187-193. 
Dempsey, D.A., Vlot, A.C., Wildermuth, M.C, and Klessig, D.F. (2011). Salicylic Acid biosynthesis and metabolism. Arabidopsis Book 9, e0156.

Endler, A., and Persson, S. (2011). Cellulose synthases and synthesis in Arabidopsis. Mol. Plant 4, 199-211.

Eulgem, T., and Somssich, I.E. (2007). Networks of WRKY transcription factors in defense signaling. Curr. Opin. Plant Biol. 10, 366-371.

Fan, M., Xu, C., Xu, K., and Hu, Y. (2012). LATERAL ORGAN BOUNDARIES DOMAIN transcription factors direct callus formation in Arabidopsis regeneration. Cell Res. 22, 1169-1180.

Florentin, A., Damri, M., and Grafi, G. (2013). Stress induces plant somatic cells to acquire some features of stem cells accompanied by selective chromatin reorganization. Dev. Dyn. 242, 11211133.

Frank, M., Guivarc'h, A., Krupková, E., Lorenz-Meyer, I., Chriqui, D. and Schmülling, T. (2002). TUMOROUS SHOOT DEVELOPMENT (TSD) genes are required for co-ordinated plant shoot development. Plant J. 29, 73-85.

Fu, X., and Harberd, N.P. (2003). Auxin promotes Arabidopsis root growth by modulating gibberellin response. Nature 421, 740-743.

Gaj, M.D., Zhang, S., Harada, J.J., and Lemaux, P.G. (2005). Leafy cotyledon genes are essential for induction of somatic embryogenesis of Arabidopsis. Planta 222, 977-988.

Garcia-Ruiz, H., Carbonell, A., Hoyer, J.S., Fahlgren, N., Gilbert, K.B., Takeda, A., Giampetruzzi, A., Garcia Ruiz, M.T., McGinn, M.G., Lowery, N., et al. (2015). Roles and programming of Arabidopsis ARGONAUTE proteins during Turnip mosaic virus infection. PLoS Pathog. 11, e1004755.

Gasciolli, V., Mallory, A.C., Bartel, D.P., and Vaucheret, H. (2005). Partially redundant functions of Arabidopsis DICER-like enzymes and a role for DCL4 in producing trans-acting siRNAs. Curr. Biol. 15, 1494-1500.

Gaspar-Maia, A., Alajem, A., Meshorer, E., and Ramalho-Santos, M. (2011). Open chromatin in pluripotency and reprogramming. Nat. Rev. Mol. Cell Biol. 12, 36-47.

Gohlke, J., and Deeken, R. (2014). Plant responses to Agrobacterium tumefaciens and crown gall development. Front. Plant Sci. 5 , 155.

González-García, M.P., Vilarrasa-Blasi, J., Zhiponova, M., Divol, F., Mora-García, S., Russinova, E., and Caño-Delgado, A.I. (2011). Brassinosteroids control meristem size by promoting cell cycle progression in Arabidopsis roots. Development 138, 849-959.

Grafi, G. (2004). How cells dedifferentiate: a lesson from plants. Dev. Biol. 268, 1-6.

Grafi, G., and Barak, S. (2015). Stress induces cell dedifferentiation in plants. Biochim. Biophys. Acta. 1849, 378-384.

Grafi, G., Chalifa-Caspi, V., Nagar, T., Plaschkes, I., Barak, S., and Ransbotyn, V. (2011). Plant response to stress meets dedifferentiation. Planta 233, 433-438.

Guo, L., Yu, Y., Law, J.A., and Zhang, X. (2010). SET DOMAIN GROUP2 is the major histone $\mathrm{H} 3$ lysine [corrected] 4 trimethyltransferase in Arabidopsis. Proc. Natl. Acad. Sci. USA 107, 18557-18562.

Guo, F., Liu, C., Xia, H., Bi, Y., Zhao, C., Zhao, S., Hou, L., Li, F., and Wang, X. (2013). Induced expression of AtLEC1 and AtLEC2 differentially promotes somatic embryogenesis in transgenic tobacco plants. PLoS One 8, e71714.

Harding, E.W., Tang, W., Nichols, K.W., Fernandez, D.E., and Perry, S.E. (2003). Expression and maintenance of embryogenic potential is enhanced through constitutive expression of AGAMOUS-LIKE 15. Plant Physiol. 133, 653-663.

He, C., Chen, X., Huang, H., and Xu, L. (2012). Reprogramming of H3K27me3 is critical for acquisition of pluripotency from cultured Arabidopsis tissues. PLoS Genet. 8, e1002911.

Henderson, I.R., Zhang, X., Lu, C., Johnson, L., Meyers, B.C., Green, P.J., and Jacobsen, S.E. (2006). Dissecting Arabidopsis thaliana DICER function in small RNA processing, gene silencing and DNA methylation patterning. Nat. Genet. 38, 721-725.

Holmes-Davis, R., Tanaka, C.K., Vensel, W.H., Hurkman, W.J., and McCormick, S. (2005). Proteome mapping of mature pollen of Arabidopsis thaliana. Proteomics 5, 4864-4884.

Huang, D.W., Sherman, B.T., and Lempicki, R.A. (2009). Systematic and integrative analysis of large gene lists using DAVID bioinformatics resources. Nature Protoc. 4, 44-57.

Ikeda-Iwai, M., Umehara, M., Satoh, S., and Kamada, H. (2003).
Stress-induced somatic embryogenesis in vegetative tissues of Arabidopsis thaliana. Plant J. 34, 107-114.

Ikeda, Y., Banno, H., Niu, Q.W., Howell, S.H., and Chua, N.H. (2006). The ENHANCER OF SHOOT REGENERATION 2 gene in Arabidopsis regulates CUP-SHAPED COTYLEDON 1 at the transcriptional level and controls cotyledon development. Plant Cell Physiol. 47, 1443-1456.

Ikeuchi, M., Sugimoto, K., and Iwase, A. (2013). Plant callus: mechanisms of induction and repression. Plant Cell 25, 31593173.

Iwase, A., Mitsuda, N., Koyama, T., Hiratsu, K., Kojima, M., Arai, T., Inoue, Y., Seki, M., Sakakibara, H., Sugimoto, K., et al. (2011). The AP2/ERF transcription factor WIND1 controls cell dedifferentiation in Arabidopsis. Curr.Biol. 21, 508-514.

Keegstra, K. (2010). Plant cell walls. Plant Physiol. 154, 483-486.

Kim, M.G., Kim, S.Y., Kim, W.Y., Mackey, D., and Lee, S.Y. (2008). Responses of Arabidopsis thaliana to challenge by Pseudomonas syringae. Mol. Cells 25, 323-331.

Kosugi, S., and Ohashi, Y. (2003). Constitutive E2F expression in tobacco plants exhibits altered cell cycle control and morphological change in a cell type-specific manner. Plant Physiol. 132, 2012-2022.

Kötting, O., Pusch, K., Tiessen, A., Geigenberger, P., Steup, M., and Ritte, G. (2005). Identification of a novel enzyme required for starch metabolism in Arabidopsis leaves. The phosphoglucan, water dikinase. Plant Physiol. 137, 242-252.

Koyama, T., Mitsuda, N., Seki, M., Shinozaki, K., and Ohme-Takagi, M. (2010). TCP transcription factors regulate the activities of ASYMMETRIC LEAVES1 and miR164, as well as the auxin response, during differentiation of leaves in Arabidopsis. Plant Cell 22, 3574-3588.

Kumar, R., Kushalappa, K., Godt, D., Pidkowich, M.S., Pastorelli, S. Hepworth, S.R., and Haughn, G.W. (2007). The Arabidopsis BEL1-LIKE HOMEODOMAIN proteins SAW1 and SAW2 act redundantly to regulate $K N O X$ expression spatially in leaf margins. Plant Cell 19, 2719-2735.

Lafos, M., Kroll, P., Hohenstatt, M.L., Thorpe, F.L., Clarenz, O., and Schubert, D. (2011). Dynamic regulation of H3K27 trimethylation during Arabidopsis differentiation. PLoS Genet. 7, e1002040.

Lee, H.G., Mas, P., and Seo, P.J. (2016). MYB96 shapes the circadian gating of ABA signaling in Arabidopsis. Sci. Rep. 6, 17754.

Mason, M.G., Mathews, D.E., Argyros, D.A., Maxwell, B.B., Kieber J.J., Alonso, J.M., Ecker, J.R., and Schaller, G.E. (2005). Multiple type-B response regulators mediate cytokinin signal transduction in Arabidopsis. Plant Cell 17, 3007-3018.

Nag, A., King, S., and Jack, T. (2009). miR319a targeting of TCP4 is critical for petal growth and development in Arabidopsis. Proc. Natl. Acad. Sci. USA 106, 22534-22539.

Nakamichi, N., Ito, S., Oyama, T., Yamashino, T., Kondo, T., and Mizuno, T. (2004). Characterization of plant circadian rhythms by employing Arabidopsis cultured cells with bioluminescence reporters. Plant Cell Physiol. 45, 57-67.

Narbonne, P., and Roy, R. (2006). Regulation of germline stem cell proliferation downstream of nutrient sensing. Cell Div. 1,29.

Nicaise, V., Roux, M., and Zipfel, C. (2009). Recent advances in PAMP-triggered immunity against bacteria: pattern recognition receptors watch over and raise the alarm. Plant Physiol. 150, 1638-1647.

Niederhuth, C.E., Patharkar, O.R., and Walker, J.C. (2013). Transcriptional profiling of the Arabidopsis abscission mutant hae hsl2 by RNA-Seq. BMC Genomics 14, 37.

Nuruzzaman, M., Sharoni, A.M., and Kikuchi, S. (2013). Roles of NAC transcription factors in the regulation of biotic and abiotic stress responses in plants. Front. Microbiol. 4, 248.

Okushima, Y., Fukaki, H., Onoda, M., Theologis, A., and Tasaka, M. (2007). ARF7 and ARF19 regulate lateral root formation via direct activation of $L B D / A S L$ genes in Arabidopsis. Plant Cell 19, 118-130.

Passaia, G., Queval, G., Bai, J., Margis-Pinheiro, M., and Foyer, C.H. (2014). The effects of redox controls mediated by glutathione peroxidases on root architecture in Arabidopsis thaliana. J. Exp. Bot. 65, 1403-1413.

Pumplin, N., and Voinnet, O. (2013). RNA silencing suppression by plant pathogens: defence, counter-defence and counter-counterdefence. Nat. Rev. Microbiol. 11, 745-760.

Ramalho-Santos, M., Yoon, S., Matsuzaki, Y., Mulligan, R.C., and 
Melton, D.A. (2002). "Stemness": transcriptional profiling of embryonic and adult stem cells. Science 298, 597-600.

Rasmussen, M.W., Roux, M., Petersen, M., and Mundy, J. (2012). MAP kinase cascades in Arabidopsis innate immunity. Front. Plant Sci. 3, 169.

Rodrigues, A., Adamo, M., Crozet, P., Margalha, L., Confraria, A., Martinho, C., Elias, A., Rabissi, A., Lumbreras, V., GonzálezGuzmán, M., et al. (2013). ABl1 and PP2CA phosphatases are negative regulators of SNF1-related protein kinase1 signaling in Arabidopsis. Plant Cell 25, 3871-3884.

Sakai, H., Honma, T., Aoyama, T., Sato, S., Kato, T., Tabata, S., and Oka, A. (2001). ARR1, a transcription factor for genes immediately responsive to cytokinins. Science $294,1519-1521$.

Santelia, D., Kötting, O., Seung, D., Schubert, M., Thalmann, M., Bischof, S., Meekins, D.A., Lutz, A., Patron, N., Gentry, M.S., et al. (2011). The phosphoglucan phosphatase like sex Four2 dephosphorylates starch at the C3-position in Arabidopsis. Plant cell 23, 4096-4111.

Sieberer, T., Hauser, M.T., Seifert, G.J., and Luschnig, C. (2003). PROPORZ1, a putative Arabidopsis transcriptional adaptor protein, mediates auxin and cytokinin signals in the control of cell proliferation. Curr. Biol. 13, 837-842.

Skylar, A., Sung, F., Hong, F., Chory, J., and Wu, X. (2011). Metabolic sugar signal promotes Arabidopsis meristematic proliferation via G2. Dev. Biol. 351, 82-89.

Smith, A.M., Zeeman, S.C., and Smith, S.M. (2005). Starch degradation. Annu. Rev. Plant Biol. 56, 73-98.

Streb, S., Eicke, S., and Zeeman, S.C. (2012). The simultaneous abolition of three starch hydrolases blocks transient starch breakdown in Arabidopsis. J. Biol. Chem. 287, 41745-41756.

Sugimoto, K., Jiao, Y., and Meyerowitz, E.M. (2010). Arabidopsis regeneration from multiple tissues occurs via a root development pathway. Dev. Cell 18, 463-471.

Tao, Q., Guo, D., Wei, B., Zhang, F., Pang, C., Jiang, H., Zhang, J., Wei, T., Gu, H., Qu, L.J., et al. (2013). The TIE1 transcriptional repressor links TCP transcription factors with TOPLESS/ TOPLESS-RELATED corepressors and modulates leaf development in Arabidopsis. Plant Cell 25, 421-437.
Thakare, D., Tang, W., Hill, K., and Perry SE. (2008). The MADSdomain transcriptional regulator AGAMOUS-LIKE15 promotes somatic embryo development in Arabidopsis and soybean. Plant Physiol. 146, 1663-1672.

Trapnell, C., Pachter, L., and Salzberg, S.L. (2009). TopHat: discovering splice junctions with RNA-Seq. Bioinformatics 25, 11051111.

Trigueros, M., Navarrete-Gómez, M., Sato, S., Christensen, S.K. Pelaz, S., Weigel, D., Yanofsky, M.F., and Ferrándiz, C. (2009). The NGATHA genes direct style development in the Arabidopsis gynoecium. Plant Cell 21, 1394-1409.

Tsukagoshi, H., Busch, W., and Benfey, P.N. (2010). Transcriptional regulation of ROS controls transition from proliferation to differentiation in the root. Cell 143, 606-616.

Tsuwamoto, R., Yokoi, S., and Takahata, Y. (2010). Arabidopsis EMBRYOMAKER encoding an AP2 domain transcription factor plays a key role in developmental change from vegetative to embryonic phase. Plant Mol. Biol. 73, 481-492.

Vanstraelen, M., and Benková, E. (2012). Hormonal interactions in the regulation of plant development. Annu. Rev. Cell Dev. Biol. 28, 463-487.

Xing, Y., Jia, W., and Zhang, J. (2007). AtMEK1 mediates stressinduced gene expression of CAT1 catalase by triggering $\mathrm{H} 2 \mathrm{O} 2$ production in Arabidopsis. J. Exp. Bot. 58, 2969-2981.

Xing, Y., Cao, Q., Zhang, Q., Qin, L., Jia, W., and Zhang, J. (2013). MKK5 regulates high light-induced gene expression of $\mathrm{Cu} / \mathrm{Zn}$ superoxide dismutase 1 and 2 in Arabidopsis. Plant Cell Physiol. 54, 1217-1227.

Yakir, E., Hilman, D., Harir, Y., and Green, R.M. (2007). Regulation of output from the plant circadian clock. FEBS J. 274, 335-345.

Yang, H., Mo, H., Fan, D., Cao, Y., Cui, S., and Ma, L. (2012). Overexpression of a histone H3K4 demethylase, JMJ15, accelerates flowering time in Arabidopsis. Plant Cell Rep. 31, 12971308.

Zuo, J., Niu, Q.W., Frugis, G., and Chua, N.H. (2002). The WUSCHEL gene promotes vegetative-to-embryonic transition in Arabidopsis. Plant J. 30, 349-359. 